\title{
Time-resolved Velocity Estimation from Inflow Pressure Measurements in a Subsonic Jet Using Machine-Learning Methods
}

\author{
Songqi $\mathrm{Li}^{1}$, Wenyan $\mathrm{Li}^{2}$, Lawrence Ukeiley ${ }^{1 *}$ \\ ${ }^{1}$ University of Florida, Gainesville, Florida, USA \\ ${ }^{2}$ Comcast Applied AI Research Lab, Washington DC, USA \\ *ukeiley@ufl.edu
}

The goal of this study is to estimate aspects of the time-resolved (TR) velocity field that is associated with pressure fluctuations measured in a subsonic jet using machine learning (ML) approaches. The experiments were conducted in the Anechoic Jet Test Facility at the University of Florida using a round converging nozzle operated at at a Mach number of 0.3 and $R e_{D}=3.8 \times 10^{5}$. Planar PIV was utilized to record nonTR, 2D velocity snapshots on the streamwise plane. A B\&K 4138 1/8" microphone and a GRAS 46DD $1 / 8$ " microphone were employed to measure inflow pressure fluctuations synchronously with the PIV. Both microphones were equipped with aerodynamically-shaped nosecones and were placed on the upper and lower jet liplines. The nosecone tips were streamwisely aligned and were placed just downstream of the PIV window (see Figure 1(a)p. Pressure signals were recorded synchronously with PIV, but at different sampling rates, $80 \mathrm{kHz}$ and $12 \mathrm{~Hz}$, respectively. A total of $8000 \mathrm{PIV}$ snapshots were acquired in the experiment.

The potential of ML-based techniques to tackle problems in fluid dynamics has been justified in [1; 2]. In this work a ML estimation approach was applied to estimate TR velocity from the experimental dataset (see Figure 1(b)]. With the application of the snapshot POD [3], the dominant spatial features of velocity were represented by the leading POD modes, which shifts the focus onto the estimation of TR-POD expansion coefficients from time-lagged pressure using proper input-output models. Two neural network (NN) architectures were proposed to implement the model. The first model is a multi-layer perceptron (MLP) with two fully connected hidden layers. The second architecture is composed of a two-layered bidirectional long-short-term-memory (Bi-LSTM) and an output layer stemming from the hidden layer output at the laser burst time. The time-dependent parameter transmission mechanism makes Bi-LSTM effective to distill information from past and future time steps. An iterative regression approach was utilized to train the models until convergence. TR-POD expansion coefficients were estimated by feeding continuous pressure signals to the trained networks, from which velocity was reconstructed in combination with the POD spatial eigenfunctions.

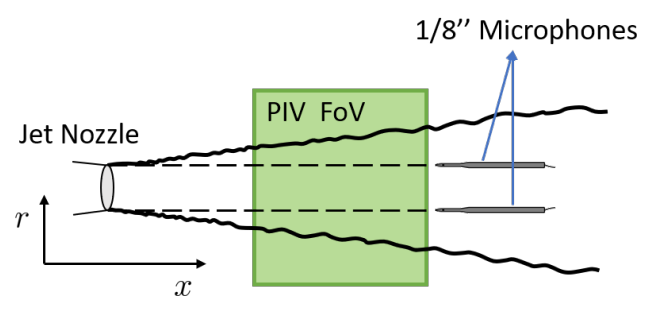

(a) Experimental setup of inflow pressure measurements synchronized with PIV
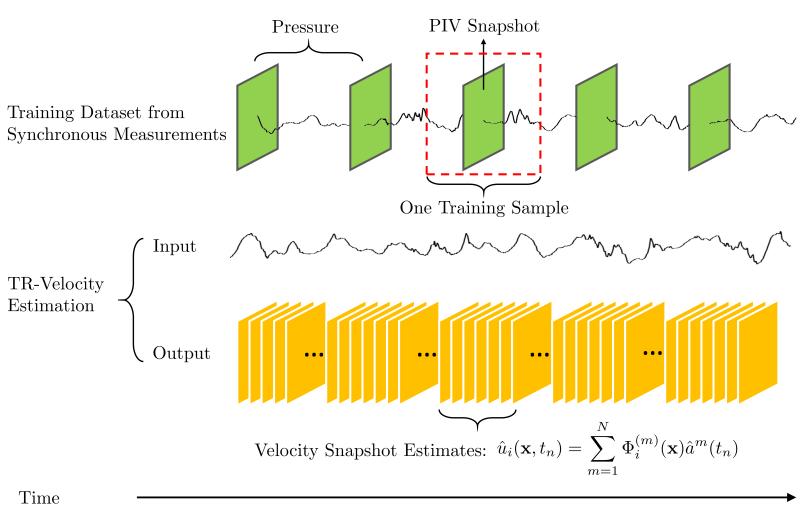

(b) Overview of velocity estimation from pressure inputs

Figure 1: Time-resolved velocity estimation from inflow pressure synchronized measured with PIV. 


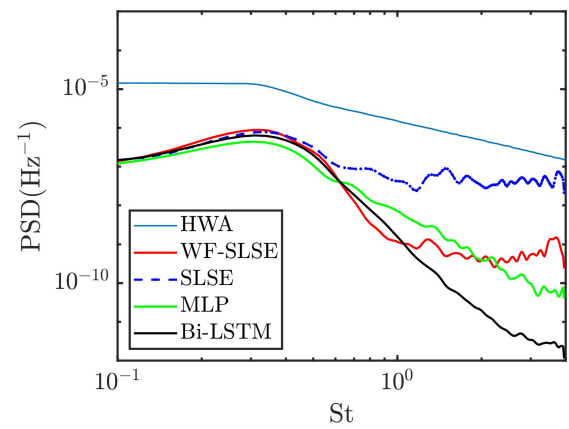

(a) $r / D=0$

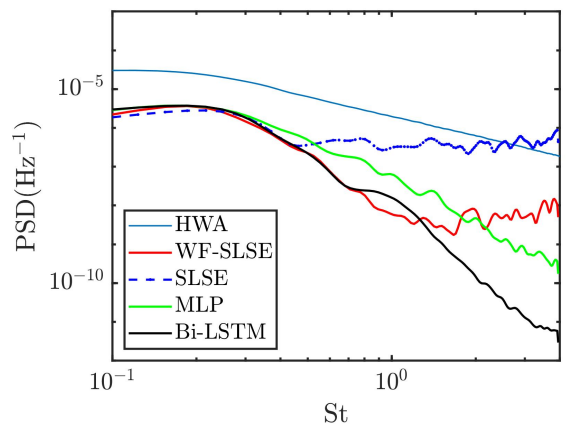

(b) $r / D=0.5$
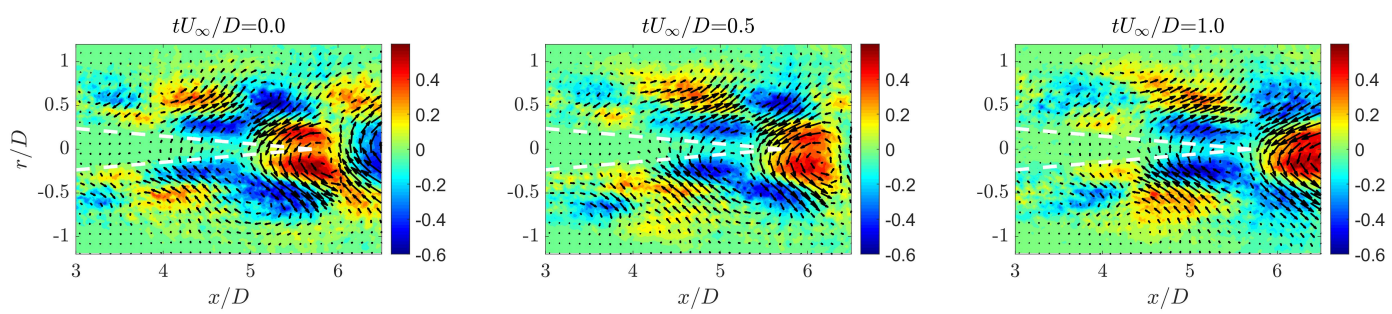

(c) real-time estimation with $2 \mathrm{D}$ vorticity $\left(\omega \frac{D}{U_{\infty}}\right)$ contours and velocity vectors

Figure 2: (a),(b): A comparison of velocity spectra at $x / D=6$ from $\mathrm{NN}$ architectures, stochastic estimation, and howtire measurements; (c): Instantaneous flow reconstruction from Bi-LSTM.

Figures 2(a) and 2(b) display the streamwise velocity spectra estimated from NN architectures using the first 50 POD modes, with the results compared to ones from Spectral Linear Stochastic Estimation (SLSE) [4] and hotwire measurements at same locations. Both NN schemes are capable of highlighting the broadband peak at low Strouhal numbers. The unique advantage of Bi-LSTM architecture is featured in the attenuation of high frequency noise with a rapid roll-off. Time-resolved velocity reconstruction from the BiLSTM architecture is exhibited in Figure 2(c), where the streamwise convection of coherent structures inside the jet mixing layer is observed as well as the formation of larger eddies downstream of the jet potential core. The estimation result represents the space-time dynamics of the acoustic sources in the jet mixing layer, and is of great importance to enhance the understanding of the noise generation mechanism.

\section{Acknowledgements}

The authors at the University of Florida acknowledge the support of NSF under award CBET-1704768.

\section{References}

[1] Mohan, A. T., and Gaitonde, D. V., "A deep learning based approach to reduced order modeling for turbulent flow control using LSTM neural networks," arXiv:1804.09269, 2018.

[2] Brunton, S. L., Noack, B. R., and Koumoutsakos, P., "Machine Learning for Fluid Mechanics," Annual Review of Fluid Mechanics, Vol. 52, No. 1, 2020, pp. 477-508.

[3] Sirovich, L., "Turbulence and the dynamics of coherent structures part I: coherent structures," Quarterly of Applied Mathematics, Vol. 45, No. 3, 1987, pp. 561-571.

[4] Tinney, C., Coiffet, F., Delville, J., Hall, A., Jordan, P., and Glauser, M., "On spectral linear stochastic estimation," Experiments in fluids, Vol. 41, No. 5, 2006, pp. 763-775. 